\title{
Investigation of Bubble Size Distributions in Oscillatory Flow at Various Flow Rates
}

\author{
A. Siswanto, D. Kuvshinov, W. B. Zimmerman \\ Chemical and Biological Engineering Department, Faculty of Engineering, University of Sheffield
}

\begin{abstract}
The demand for eco-friendly technologies for industrial processes is increasing. Various factors such as labour costs, exploitation and waste management influences the production costs and hence the profit generated. The water treatment industry is one example of a well-developed sector which is facing these concerns. Microbubble techniques exhibit many benefits which make them applicable to heterogeneous processes, such as longer contact time and greater surface area giving higher mass transfer rates. The Fluidic Oscillator (FO) is a relatively new device which enhances the efficacy of microbubble generation. The FO produces smaller bubbles by applying oscillatory flow through a diffuser to generate microbubbles. This paper aims to study the influence of steady and oscillatory flow to bubble size distribution. The experimentation was carried out with a ceramic diffuser to produce microbubbles in water. A High Speed Camera was used for bubble imaging. Data on bubble distributions at various flow rates was obtained by image post-processing. It was observed that the application of the FO at selected operational conditions gave narrower bubble size distributions in a range of flow rates compared to continuous flow. The range of applications of this technology is continuously increasing.
\end{abstract}

Keywords Bubble Distribution; Ceramic Diffuser; Fluidic Oscillator; Microbubbles; Steady Flow

\section{INTRODUCTION}

A significant number of industrial processes include water in the production chain. Nowadays requirements for water recycling and treatment are stricter which results in higher production costs. It is important to apply modern approaches to water treatment to reduce the final cost of products.

Microbubble technology has been recognised over past decades as a prospective root for intensification of water treatment processes. There is no agreement yet between researchers about the size range for microbubbles but it is currently defined as bubbles which have diameter less than $1000 \mu \mathrm{m}$. There are various ways to produce microbubbles. A set of methods for microbubble generation including nozzles, membrane and gas spargers have been discussed [1]. Microbubbles offer many advantages; in particular: (a) smaller size of bubbles giving larger contact area, (b) greater mass transfer in gas-liquid interface due to larger contact area and (c) longer contact times between gas and liquid due to slower rising velocity [2]. Furthermore, application of microbubbles is effective in combination with other chemical processes, i.e. ozone treatment [3] and other liquid based processes [4].

A fluidic oscillator (FO) is a microbubble generator which offers low power consumption with bubble size as desired (5). The basic principle of FO is the oscillation of air flow. It consists of non-moving parts with a feedback loop attached. The compact and robust design of FO makes it applicable for industry plants of different scales.

The aim of this research is to characterise the bubble formation process with the use of a ceramic diffuser at steady flow using a FO.

\section{MATERIALS AND METHODS}

The experimentation was done by visualisation of bubble formation through a ceramic diffuser with an average pore size of $20 \mu \mathrm{m}$, made from porous alumina: silica (80\%:20\% $\mathrm{w} / \mathrm{w})$. Three different flow rates (1 lpm, $2 \mathrm{lpm}$, and $3 \mathrm{lpm})$ were set as air flow rate through the diffuser. Fig. 1 presents an overview of the bubble formation process during the experimentation.

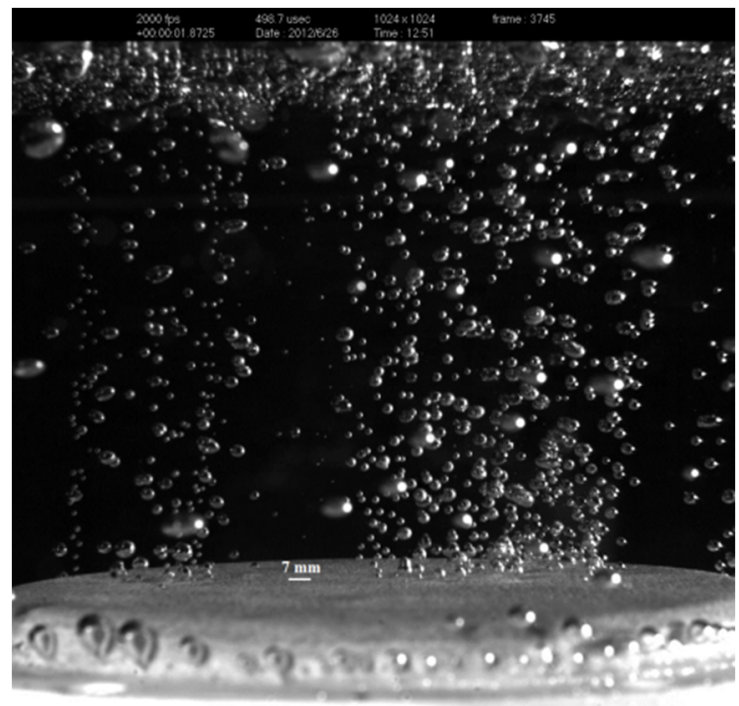

Figure 1. Microbubble production, overview.

A FASTCAM SA3 Photron, 120K-M3 high speed camera was used for imaging generated microbubbles. The camera was computer controlled by the PFV Photron FASTCAM software. A Tesař-Zimmerman fluidic oscillator was applied in order to get oscillatory flow in the set up.

\section{RESULTS AND DisCUSSIONS}

The effect of oscillated flow was examined by comparing bubble diameters produced for each flow type at different flow rates. Fig. 2 and Fig. 3 shows the corresponding data. 


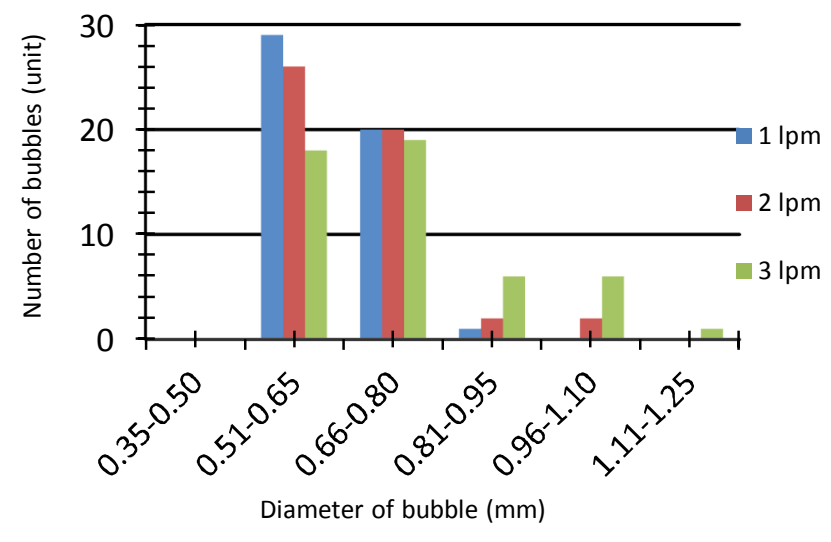

Figure 2. Bubble size distributions in steady flow.

Fig. 2 presents the bubble size distribution without the presence of a FO. At low flow rates of $1 \mathrm{lpm}$, the greatest frequency of bubble diameters was between 0.51-0.65 mm. The same was true of medium flow rates, $2 \mathrm{lpm}$. However, the bubbles produced at high flow rates, $3 \mathrm{lpm}$, had the highest frequency from 0.66 to $0.80 \mathrm{~mm}$. In terms of bubble size distribution, a narrow distribution was found at low flow rates, with wider distribution at high flow rates. This phenomenon is in agreement with the fact that a higher gas velocity results in lower contact time between the bubbles and the surface of the diffuser. Therefore bubbles at low flow rates were detaching more slowly once they were formed.

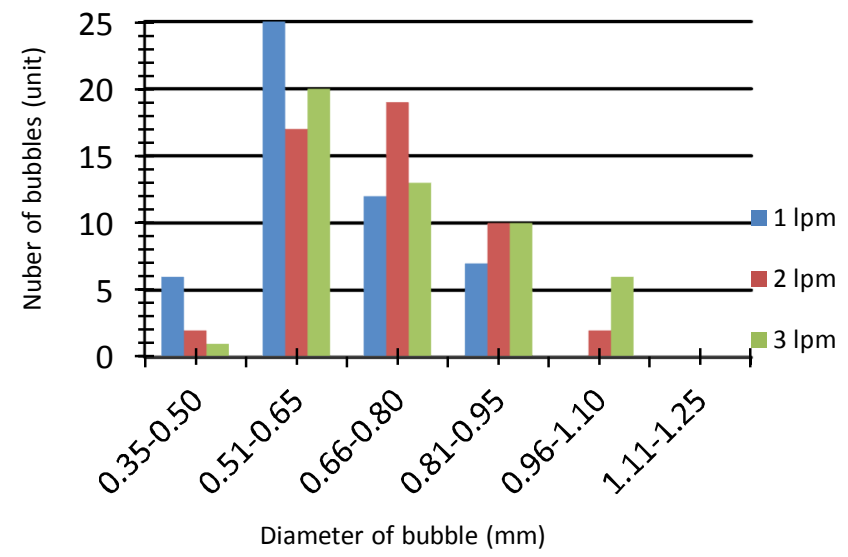

Figure 3. Bubble size distributions in oscillatory flow.
Fig. 3 describes the bubble size distribution with the presence of a FO. More bubbles were produced in the smaller range, $0.35-0.50 \mathrm{~mm}$, for low (1 lpm), medium (2 Ipm) and high flow rates ( $3 \mathrm{lpm})$, respectively. The bubble size distributions differed in that wider distribution occurring at a medium flow rate, $2 \mathrm{lpm}$. Moreover, a narrower distribution developed at a higher flow rate of $3 \mathrm{lpm}$, in comparison to bubbles size distributions obtained without the presence of a FO.

\section{CONCLUSIONS}

It is shown that the presence of a FO has significant influence on microbubble generation throughout the entire range of flow rates applied. There is a $20 \%$ improvement in the produced bubbles size with the application of a FO. Investigation of FO utilisation at higher flow rates is being carried out. It aims to meet the industrial needs of FO scale up.

\section{ACKNOWLEDGEMENTS}

We gratefully acknowledge EPSRC for offering support to Microbubbles Programme in terms of imaging capabilities. AS would also like to acknowledge The Directorate General of Higher Education Indonesia, Ministry of Education and Culture of the Republic of Indonesia, and the Education Attaché, the Embassy of the Republic of Indonesia in United Kingdom for her doctoral scholarship.

\section{REFERENCES}

1. Li P. Development of Advanced Water Treatment Technology Using Microbubbles Keio University, Japan; 2006.

2. Parmar R, Majumder SK. Microbubble Generation and Microbubble-aided Transport Process Intensification-A stateof-the-art Report. Chemical Engineering and Processing: Process Intensification. 2013;64:79-97.

3. Kuvshinov D, Siswanto A, Zimmerman WB. Microbubbles Enhanced Synthetic Phorbol Ester Degradation by Ozonolysis. International Journal of Chemical, Materials Science and Engineering. 2014;8(1):78-81.

4. Agarwal A, Jern Ng W, Liu Y. Principle and Applications of Microbubble and Nanobubble Technology for Water Treatment. Chemosphere. 2011;84:1175-80.

5. Zimmerman WB, Tesar, V., Butler, S., Bandulusen, H.C.H. Microbubble Generation. Recents Patents on Engineering. 2008;2(1):1-8. 